\title{
REVIEW
}

www.nature.com/hdy

\section{Recent developments in genetic data analysis: what can they tell us about human demographic history?}

\author{
MA Beaumont \\ School of Animal and Microbial Sciences, University of Reading, Whiteknights, PO Box 228, Reading RG6 6AJ, UK
}

\begin{abstract}
Over the last decade, a number of new methods of population genetic analysis based on likelihood have been introduced. This review describes and explains the general statistical techniques that have recently been used, and discusses the underlying population genetic models. Experimental papers that use these methods to infer human demographic and phylogeographic history are reviewed. It appears that the use of likelihood has hitherto had little impact in the field of human population genetics, which is still
\end{abstract}

primarily driven by more traditional approaches. However, with the current uncertainty about the effects of natural selection, population structure and ascertainment of singlenucleotide polymorphism markers, it is suggested that likelihood-based methods may have a greater impact in the future.

Heredity (2004) 92, 365-379, advance online publication, 24 March 2004; doi:10.1038/sj.hdy.6800447

Keywords: human evolution; coalescent; likelihood; population structure; admixture; population growth

\section{Introduction}

The aims of this article are to give an overview of modelbased, primarily genealogical, statistical methods that have recently been developed for population genetic analysis, and then to discuss a number of applications to human populations. Given this perspective, it does not aim to be an exhaustive treatment of the genetic evidence for past human demographic history, which has been well covered recently (Harpending and Rogers, 2000; Relethford, 2001; Goldstein and Chikhi, 2002; CavalliSforza and Feldman, 2003). The motivation behind this review is that the connection between genetic data and demographic history appears rather more complicated than hitherto appreciated, and, as evidence of the widespread effects of selection in the human genome continues to mount (Przeworski et al, 2000; Nachman, 2001; Payseur and Nachman, 2002; but see Hellmann et al, 2003), and with increasing concerns of the subtle effects of ascertainment and population structure (Wakeley, 1999; Wakeley et al, 2001; Ptak and Przeworski, 2002), it is from a rigorous model-based perspective that progress may be best made. Humans are an ideal model organism for this research programme because of the wide availability of genetic markers, background historical information and general availability of funding. Information obtained from studies of humans can then help us to understand evolutionary processes in other organisms. For modellers, human data sets are commonly used as example applications to illustrate the use of their methods. In contrast, however, both in human and nonhuman studies, a current trend in empirical papers appears to be more towards qualitative analyses based on genetic trees or networks, obtained using standard phylogenetic methods. Obviously such an approach, entailing a close mapping between the shape of a genetree and demographic or phylogeographic history, is superficially very attractive and appeals to common sense, but there is a danger that it might tend towards subjectivity (Hey and Machado, 2003). There is a need therefore for greater bridge building between modellers and empiricists, and this review is a modest attempt at helping to lay a few stones in this direction.

\section{Methods of statistical analysis}

The most common approach to data analysis in population genetics has been to derive expectations of summary statistics as a function of the demographic and mutational parameters, replacing the expected value by that computed from data, and then estimating the parameter(s). An example of this is estimation of the scaled mutation rate $\theta=4 N \mu$ from the heterozygosity, $H$. Heterozygosity can be regarded as an average distance between pairs of genes, such that they have a distance 0 if they are the same and 1 if they are different. It can be calculated as $H=n /(n-1)\left(1-\sum x_{i}^{2}\right)$. Under the infinite allele model, where each new mutation is unique, the expected value, $E[H]$, is the probability that two genes have at least one mutation in either of the two lineages since their common ancestor. From coalescent theory (see, eg, Hudson, 1991), looking backwards in time, the rate at which two lineages coalesce to form a common ancestor is $1 /(2 N)$, and the rate that mutations occur along either of the two lineages is $2 \mu$. So, the probability that two genes have at least one mutation in their descent from their common ancestor is the probability that a 
mutation occurs before a coalescence, looking backwards in time, and is given by the ratio of mutation rate to total rate $2 \mu /(2 \mu+1 /(2 N))=4 N \mu /(1+4 N \mu)$. To infer $\theta$, we 'pretend' that the sample heterozygosity is the same as the expected heterozygosity, and we invert this relationship to estimate $\hat{\theta}=H /(1-H)$. Confidence intervals can either be calculated from sampling theory, or, more generally by simulation from the appropriate model. Alternatively, the distribution of the summary statistic under a suitable null hypothesis can be obtained by simulation, enabling tests of significance to be carried out. These methods are generally best for simple oneparameter models, and inferences generally have to be conditional on the values of other parameters. These methods have been the mainstay of population genetics, and it would be fair to say that most advances in the understanding of the past evolutionary history of populations have come from the application of these methods to the huge amount of new molecular data that are being collected.

The recent interest in likelihood and probabilistic modelling stems primarily from the use of computationally intensive sampling methods, which became feasible for many problems, once computers were sufficiently fast. This appears to have occurred in the statistical literature in the early 1990s, and has rapidly transferred into population genetics. Following this use, there has also been a concomitant rise in interest in Bayesian inference (Box 1; see also Shoemaker et al, 1999). To illustrate the different sampling methods (which are useful for any likelihood-based analysis, not only Bayesian), I will use an example suggested by Stephens and Donnelly (2000; see also Stephens, 2001). Given a data set $D$, there is some unknown genetic history, $G$, that gave rise to it. The genetic history broadly consists of the gene genealogy (gene 'tree') and the mutations that have

\section{Box 1}

In Bayesian analysis one assumes effectively a 'many worlds' hypothesis in which the data and parameter values are random variables that exist on an equal footing. Thus, there is a joint probability distribution $P(D, \Phi)$ giving the frequency with which a particular set of parameter values, $\Phi$, and set of data, $D$, are observed together. Once a particular data set is observed, this restricts the probability distribution of the parameters to that conditional on the data, denoted by $P(\Phi \mid D)$, which is

$$
P(\Phi \mid D)=\frac{P(D, \Phi)}{P(D)} .
$$

$P(D)$ is the marginal probability of the data - that is, the probability of the data irrespective, of the parameter values. Obviously, $P(D, \Phi)$ does not exist in vacuo, and has to be created from a model. Since

$$
P(\Phi \mid D) P(D)=P(D, \Phi)=P(D \mid \Phi) P(\Phi)
$$

$P(D, \Phi)$ is conveniently obtained from the product of the likelihood, $P(D \mid \Phi)$, and the prior, $P(\Phi)$. The likelihood is a function giving the probability or frequency with which different data sets would occur for a particular set of parameter values, and the prior is a function that gives the probability or frequency with which different parameter values might occur, irrespective of (or prior to seeing) the data. Both these functions are chosen by the modeller on the basis of background information to give $P(D, \Phi)$.

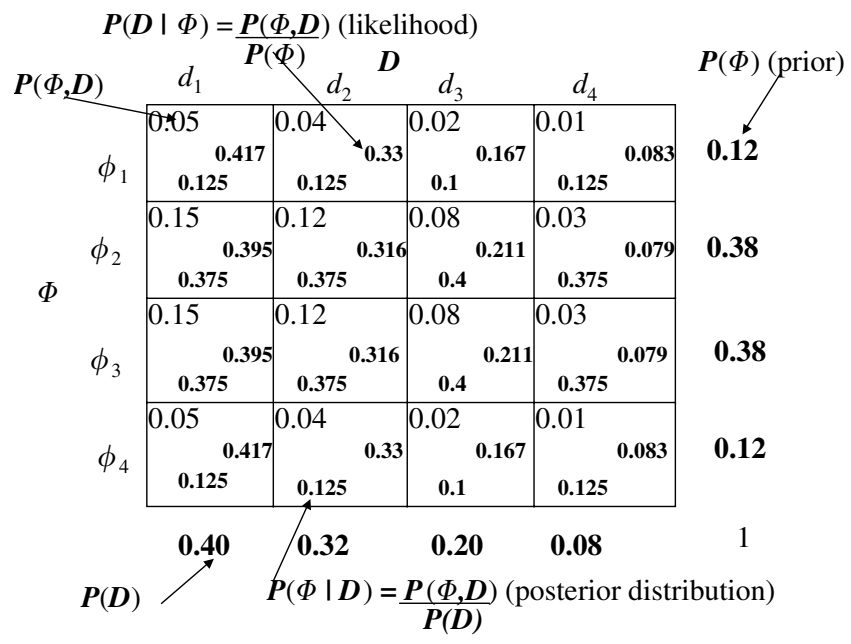

The meaning of these terms is illustrated numerically in the figure. Here I consider a data set, $D$, that can take four values $\left(d_{1}, d_{2}, d_{3}, d_{4}\right)$, and a parameter, $\Phi$, that can take four values, $\left(\phi_{1}, \phi_{2}, \phi_{3}, \phi_{4}\right)$. The table gives the joint probability with which the 16 combinations of parameter and data can occur. The marginal probabilities, $P(\Phi)$ and $P(D)$, are the sums of the joint probabilities along rows or columns and are given in the margins of the table (hence the name). The conditional probabilities - the likelihood and posterior distribution - which sum to one across their respective rows or columns, are given in the table at positions that line up with the corresponding marginal probabilities from which they were calculated. Thus in this particular example, given our model, and on observing data set $d_{3}$, we would infer that the parameter had value $\phi_{2}$ with probability 0.4 .

Often, we are dealing with complicated models with many parameters, and a key feature of the Bayesian approach (also available for a more purely likelihood-based approach with uniform priors) is that it is natural to make probability statements about particular parameters or (usually at most) a pair of parameters marginal to (ie summed/integrated over) the other parameters. For example, the above table could be viewed as the margin of a higher dimension table corresponding to other parameters, the probabilities of whose values have been summed along this extra dimension. 
occurred down the ancestral lineages since the most recent common ancestor. In a model, this genetic history could be generated, for example, by a coalescent simulation (Hudson, 1991). The genetic history, and therefore the data set, depends both on past demography - changes in reproductive patterns, population size, migration, extinction, colonisation, etc - and also on the pattern and rate of mutation for the marker that is studied. The problem from the point of view of inference is that although it is generally quite straightforward to calculate the probability $P(G \mid \Phi)$ of generating any particular genetic history, given the parameter values $\Phi$, there are very many histories that could have given rise to the data. Thus the probability of the data takes the following form:

$$
P(D \mid \Phi)=\int P(D \mid G) P(G \mid \Phi) d G
$$

where the integral denotes a summation over all possibilities, both discrete and continuous. The term $P(D \mid G)$ refers to the probability of obtaining the data given a particular genetic history. For example, if the genetic history consists of the sequence of coalescent and mutation events in the genealogy, then $P(D \mid G)=1$ if this sequence ends in the data and 0 otherwise. Although for ease of presentation, I have considered a specific representation for the genetic history, there are many other representations used for population genetic inference (eg Wilson and Balding, 1998; Felsenstein et al, 1999; Markovtsova et al, 2000). A particularly important representation is where the probability of the data is calculated, given the genealogy, marginal to the mutational history. The method for doing this is via the pruning algorithm of Felsenstein (1981), which requires some transition matrix giving the rate of change from one mutational state to another. An example of its use in genealogical inference is given in Drummond et al (2002). It should also be noted that the genetic history need not be given in a genealogical framework, and it can be computationally convenient to use, for example, the Wright-Fisher model, which describes the genetic history of the entire population (Williamson and Slatkin, 1999; Anderson et al, 2000; Wang, 2001). However, the underlying principles are the same for all these models (Felsenstein et al, 1999; Stephens and Donnelly, 2000; Stephens, 2001), and I will hereon equate the genetic history with the genealogical history from some coalescent-based model. In some simple cases, $P(D \mid \Phi)$ can be calculated straightforwardly. In general, however, Monte Carlo sampling approaches have to be used.

This simplest approach is that of Monte Carlo integration, where we simulate sample genealogical histories $G_{i} \sim P(G \mid \Phi)$ using the coalescent, and then estimate

$$
\tilde{P}(D \mid \Phi)=\sum_{i} P\left(D \mid G_{i}\right)
$$

The problem with this approach is that, in general, most simulated genealogical histories do not give rise to the data, and so the method will usually fail, although for microsatellites evolving according to the stepwise mutation model the method can be used as a means of checking other approaches for sample size up to around 10 (Beaumont, 1999).

What is required is some way of targeting the Monte Carlo integration. For example we are considering that we want $P(D \mid G)$ to be always 1 , but also we want to sample highly probable genealogical histories, the given data. To this end, we use an importance sampling function $Q(G \mid \Phi)$ from which genealogical histories are drawn. Equation (1) can be rewritten trivially as

$$
P(D \mid \Phi)=\int P(D \mid G) \frac{P(G \mid \Phi)}{Q(G \mid \Phi)} Q(G \mid \Phi) \mathrm{d} G
$$

Following the same Monte Carlo principle as above, we can then simulate $G_{i} \sim Q(G \mid \Phi)$ and estimate

$$
\tilde{P}(D \mid \Phi)=\sum_{i} P\left(D \mid G_{i}\right) \frac{P\left(G_{i} \mid \Phi\right)}{Q\left(G_{i} \mid \Phi\right)}
$$

Providing the importance sampling function is chosen well, this approach can greatly improve efficiency in the estimation of the likelihood. This method of analysis was introduced by Griffiths and Tavaré (1994). A related approach is that of Kuhner et al (1995), which is discussed in Felsenstein et al (1999) and Stephens (2001). The method of Griffiths and Tavaré (1994) has been improved in Stephens and Donnelly (2000), who have suggested an alternative importance sampling function. It is also possible to improve efficiency by resampling from the genealogical histories (see Chen and Liu, 2000).

An alternative to these sampling approaches is the use of Markov chain Monte Carlo (MCMC). This method is best considered from the point of view of Bayesian inference, in which it is most commonly used. Bayesian inference is described in Table 1, which gives the terminology used here. Essentially, MCMC draws autocorrelated random samples from the posterior distribution $P(G, \Phi \mid D)$, which is the conditional distribution of parameter values and genealogical histories given the data. The basic approach, which uses MetropolisHastings sampling, is as follows. Starting with any $G_{i}$ such that $P\left(D \mid G_{i}\right)=1$, make modifications $G_{i} \rightarrow G_{i+1}$, and $\Phi_{i} \rightarrow \Phi_{i+1}$ in such a way that it is straightforward to calculate the probability $P\left(G_{i+1}, \Phi_{i+1} \mid G_{i}, \Phi_{i}\right)$ of obtaining $G_{i+1}$ and $\Phi_{i+1}$, given that you start at $G_{i}, \Phi_{i}$, and the probability of getting back $P\left(G_{i}, \Phi_{i} \mid G_{i+1}, \Phi_{i+1}\right)$. Then accept $G_{i+1}$ and $\Phi_{i+1}$, with probability

$$
\min \left(1, \frac{P\left(G_{i+1} \mid \Phi_{i+1}\right)}{P\left(G_{i} \mid \Phi_{i}\right)} \times \frac{P\left(G_{i}, \Phi_{i} \mid G_{i+1}, \Phi_{i+1}\right)}{P\left(G_{i+1}, \Phi_{i+1} \mid G_{i}, \Phi_{i}\right)} \times \frac{P\left(\Phi_{i+1}\right)}{P\left(\Phi_{i}\right)}\right)
$$

Otherwise $G_{i+1}=G_{i}$ and $\Phi_{i+1}=\Phi_{i}$. Often, the Hastings term will be 1 because the proposed changes are symmetrical.

Provided that all the modifications are reversible, and that starting from any genealogical history and parameter values all other genealogical histories and parameter values can be reached by this Metropolis-Hastings sampling procedure, then the simulated sequence of $G_{i}$ and $\Phi_{i}$ will be an autocorrelated random sample from the Bayesian posterior distribution $P(G, \Phi \mid D)$. It is particularly convenient for Bayesian inference because it allows easy estimation of the marginal distributions (see Box 1) of particular parameters, such as growth rate, or the marginal distributions of genealogical variables of interest, such as the time until the most recent common ancestor, $T_{\text {MRCA }}$. Thus the MCMC output is a matrix of items with the rows corresponding to iterations of the MCMC. The marginal posterior distribution for a particular parameter, such as growth rate, or for a 
genealogical variable such as the $T_{\text {MRCA }}$ can be summarised using these simulated values. For example, point estimates such as the posterior mean, median or mode can be obtained - the latter corresponding to the maximum-likelihood estimate if flat priors are used. Interval estimates such as the 0.025 and 0.975 quantiles can be calculated. The distribution can be approximated or visualised, in the simplest case, by a histogram, or by kernel density estimation or local-likelihood estimation, procedures for which are available in most statistical packages.

It is difficult to make a clear distinction between what is an IS and an MCMC method, because many algorithms use aspects of both. The method of Kuhner et al (1995) uses MCMC to sample genealogical histories conditional on the data and on a particular parameter value, and this sample is then used in an IS calculation to estimate the relative likelihood for other parameter values. Another method described in Beaumont (2003) uses IS to generate independent genealogical histories, but embeds this in an MCMC simulation. In this review, an 'MCMC method' is one that gives an autocorrelated sample of parameter values from a posterior distribution, whereas an 'IS method' uses a sample of genealogical histories to compute (relative) likelihoods.

A particular feature of population genetic models is that the information in the data arises from the relative rates of different processes - mutations, coalescences, recombinations (if included in the model) - and it is these relative rates that are affected by the demographic history. Thus the number of parameters that can be independently estimated is generally one less than the total, and the models are parameterised with scaled composites such as $\theta=4 N_{e} \mu$. The typical workaround for estimating demographic parameters has been to apply a point estimate of, for example, the mutation rate or time when a population split. An advantage of the Bayesian approach is that it allows uncertainty to be incorporated into these point estimates, and reflected in the posterior distribution. Also, priors can be put on all the parameters, so that not all the weight rests with, for example, a point estimate of the mutation rate. In this way, it is natural to make inferences about all the parameters directly, rather than scaled in terms of each other.

As stated above, the driving goal behind the development of these methods is that it may be possible to obtain more precise estimates of demographic parameters if we can use all the information in the data rather than from summaries (Felsenstein, 1992). However, another very important motivation is to be able to handle multiparameter models as efficiently and flexibly as possible. This flexibility has motivated another area of current interest, which aims to obtain approximate likelihoods and posterior distributions from summary statistics rather than the data. This inevitably means that information is lost, but it still retains the benefit of flexibility. The approach is best explained from the Bayesian perspective first introduced by Pritchard et al (1999).

Referring to Table 1, it can be seen that one way to estimate the posterior distribution in this simple example is to simulate samples from the joint density $P(\Phi, D)$ by first simulating from the prior $\Phi_{i} \sim P(\Phi)$, and then simulating from the likelihood $D_{i} \sim P\left(D \mid \Phi_{i}\right)$. The posterior distribution $P(\Phi / D)=P(D, \Phi) / P(D)$ for any given $D$ and $\Phi$ can be obtained by the proportion of all simulated points that correspond to that particular $D$ and $\Phi$ divided by the proportion of points corresponding to $D$ (ignoring $\Phi)$. In the approximate approach, the data, $D$, are replaced by summary statistics, $S$, calculated from the data and we are now interested in estimating the posterior distribution $P(\Phi \mid S)$, and the same simulation method as described above is used. In general, unlike the example in Box 1, the parameters and summary statistics will be more or less continuously distributed. To accommodate this, Pritchard et al (1999) suggest accepting simulated points that have summary statistics within a region sufficiently close to those observed in the real data. However, this is essentially a problem of density estimation and other methods are available (Beaumont et al, 2002; see also Marjoram et al, 2003). A particular advantage of the summary-statistic approach over other methods, such as importance sampling or MCMC, is that it is not necessary to specify a likelihood function explicitly - there is no need to specify the likelihood function when writing a program to simulate data. In addition, the data need not only be purely genetical but also ecological observations, such as mark-recapture information, or ages of individuals, can be included into the suite of summary statistics. One merely needs a computer program for simulating genetical and/or ecological data sets. Clearly, the existence of a probabilistic simulation that generates data implies that there is some likelihood function that could be obtained. However, this may be very difficult. The attraction for biologists is that many such simulations are now available from the worldwide web, or, for example, an individual-based program can be straightforwardly written in an intuitive way, and therefore this approach is very easy to adopt with minimal statistical expertise, and should further enhance the recent inroads made by Bayesian statistics into population genetics and ecology. Examples of the application of the method under complex demographic scenarios are Estoup et al (2001) and Estoup and Clegg (2003).

\section{Models}

The main models that have so far been considered are illustrated in Figure 1. I will not consider recombining markers here, because, although potentially of great importance, model-based methods that use linked markers to study aspects of human population history are still in their infancy, and the problems of modelling have been recently exacerbated by the discovery of a large variation in the recombination rate along the genome and appreciation of the possible ubiquity of gene conversion at fine scales (see Nordborg and Tavaré, 2002, for a recent review; and see Clark et al, 2003, for discussion of the many complex issues involved). The primary workhorse has been the coalescent (Hudson, 1991; Donnelly and Tavaré, 1995; Nordborg, 2001; Rosenberg and Nordborg, 2002). This has been used to infer changes in past population size, and patterns of gene flow. In normal usage, the coalescent describes the genealogical history of a population back until the MRCA (Figure 1a). However, a simpler alternative is to consider a truncated genealogy as illustrated in Figure 1b, where the more recent genealogy is modelled without mutations from the present back until some critical time, 

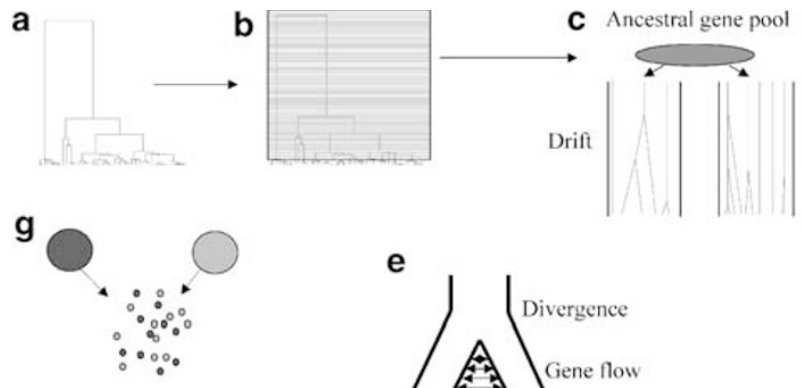

f

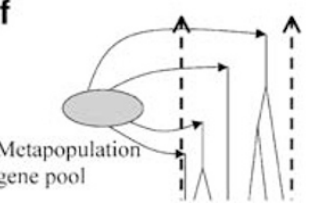

e

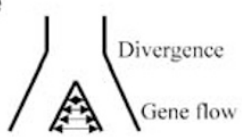

Figure 1 Examples of demographic and genealogical models that have recently been studied using likelihood-based methods. (a) A simulated genealogy of a sample of 50 chromosomes under the standard coalescent model. (b) Upper part of the genealogy is replaced by a 'gene pool' from which the lineages in the lower part are sampled. (c) An application of the model in (b) where an ancestral population splits into two, which then diverge through genetic drift. The time is assumed to be short enough that a negligible number of mutations occur in the genealogy since the time of divergence. (d) A model of admixture, where the founders of a new population comprise a mixture of individuals from two populations with different gene pools, similar to those considered in (b) and (c). After the admixture event, the three populations diverge through genetic drift. (e) A model of divergence similar to (c), but where the genealogy with mutations is fully modelled in both the ancestral and the derived populations. Gene flow continues to occur since the time of divergence. (f) A genealogy consistent with the infinite island model. Lineages coalesce, and at some point back in time are immigrants with allelic types drawn at random from the metapopulation gene pool. (g) A genotypic model where a sample consists of a mixture of individuals whose multilocus genotype is drawn at random from either one or the other parental population gene pools.

and then the lineages that have not coalesced by this time are randomly drawn from some baseline frequency distribution (Nielsen et al, 1998; O'Ryan et al, 1998; Saccheri et al, 1999). Obviously, such a model requires some way of estimating the baseline frequencies, and is well suited to study fragmented populations diverging through pure genetic drift (Figure 1c). Related to this is the situation where an admixed population is formed from founders that are derived from two independent baseline populations as illustrated in Figure 1d (Thompson, 1973; Chikhi et al, 2001; Wang, 2003). Likelihoodbased inference can also be carried out for the population divergence model with mutations throughout the genealogy (Nielsen, 1998), and this can be generalised to also include estimation of migration rates since the time of splitting (Figure 1e) (Nielsen and Wakeley, 2001).

Another 'mutation-free' model, similar to the drift model considered above, is that of the infinite-island model (Wright, 1931; Rannala and Hartigan, 1996; see reviews in Rousset, 2001 and Balding, 2003). Here, looking backwards in time, lineages coalesce or migrate out of the population at a rate proportional to the number of lineages in the genealogy (Figure 1f). The allelic type of these lineages is drawn from some baseline frequency distribution, as in the drift example above. In this case, the likelihood is given by a multinomial-
Dirichlet distribution, which can be obtained from a diffusion approach (Wright, 1951; Rannala and Hartigan, 1996). The same likelihood function can also be explicitly obtained from a coalescent argument based on a continent-island (equivalently, island-mainland) model (Balding and Nichols, 1994, 1995; Balding, 2003). The Dirichlet distribution is the multivariate counterpart to the beta distribution. In this specific case, it describes the variability of the subpopulation gene frequencies from the baseline gene frequencies as a function of the immigration rate. When the immigration rate is high, the subpopulation gene frequencies are the same as the baseline frequencies. When the immigration rate is low, there is much more variability because of drift. For given subpopulation gene frequencies, the frequency distribution in a sample is given by a multinomial distribution, and the multinomial-Dirichlet is the multinomial distribution integrated over all the possible subpopulation frequency distributions, given by the Dirichlet. Although the likelihood can be compactly expressed, computerintensive methods are required to integrate over unknown baseline gene frequencies (Balding and Nichols, 1997; Holsinger, 1999; Holsinger et al, 2002).

Related to this are a class of 'separation of time scale' models studied by Wakeley (2001). The essential idea is that when there are a large number of subpopulations, with immigrants from a migrant pool derived from all the subpopulations, the genealogical process can be divided into two phases: an initial 'scattering phase', which is the same process considered by Balding and Nichols (1994), where lineages either coalesce within subpopulations or migrate out into other subpopulations. If there are a large number of subpopulations, each migrant lineage will be in a separate subpopulation, and in this case the genealogical process can be approximated by the standard coalescent on a time scale that depends on the details of the metapopulation structure. This process has been described by Wakeley as the 'collecting phase'. The difference between this model and that underlying the use of the simple multinomial-Dirichlet formula discussed above is in the priors assumed. In the case considered earlier, some prior has to be considered for the baseline frequencies, and often a uniform Dirichlet is assumed, where all frequency distributions are equally likely. This, in fact, corresponds to a very specific mutation model with a particular mutation ratea $K$-allele mutation model where, each time a mutation occurs, it has a $1 / K$ chance of being any of the $K$ types, and where the scaled mutation rate, $\theta$, is equal to $K$ (see, eg, Griffiths and Tavaré, 1994). In contrast, the prior for the model considered here comes from an evolutionary model that can be modified to fit the particular markers under study.

More recently, there has been interest in nongenealogical genotypic models for estimating recent gene-flow, admixture, and to detect hybrid individuals (Rannala and Mountain, 1997; Pritchard et al, 2000a; see Beaumont, 2001, for a review of the history of this approach). An example of the type of model considered is illustrated in Figure $1 \mathrm{~g}$ where individuals in a sample, whose provenance is unknown, comprise a mixture of individuals from two different populations. Assuming equilibrium and random mating in the progenitor populations, and a difference in gene frequency between them, it is possible to infer the parameters governing the 
composition of the sample. An interesting feature of these genotypic methods is that extensions beyond simple assignment of individuals to populations identified beforehand has only been possible by taking a Bayesian approach and using MCMC methods (Pritchard et al, 2000a).

\section{Applications}

\section{Population growth}

Interest in using genetic information to infer patterns of human growth began in the early 1990s, stemming primarily from the confluence in the development of methods of analysis based on the coalescent (Slatkin and Hudson, 1991; Rogers and Harpending, 1992), the availability of mtDNA sequence data (Cann et al, 1987) and palaeontological evidence supporting the 'out-ofAfrica' demographic scenario (Stringer and Andrews, 1988). Thus most of the early analyses were carried out with mitochondrial sequences, using estimators based on distributions of pairwise differences between sequences ('mismatch distributions') (Rogers, 1995; Excoffier and Schneider, 1999). These studies have all tended to support recent population growth strongly.

Examples of likelihood-based methods to study population growth that have been developed are the importance sampling method of Griffiths and Tavaré (1994), available in the program Genetree (http:// www.stats.ox.ac.uk/mathgen/software.html), the importance sampling method of Kuhner et al (1998) available in the program Fluctuate (http://evolution.genetics.washington.edu/lamarc.html), the summary-statistic method of Pritchard et al (1999) for linked microsatellite data, the MCMC-based method of Beaumont (1999) for unlinked microsatellite data available in the program Msvar (http://www.rubic.rdg.ac.uk/ $\sim$ mab) and, for linked microsatellite data, the MCMCbased method of Wilson et al (2003) (http://www.maths/ abdn.ac.uk/ ijw), developed from Wilson and Balding (1998). An MCMC method, based on pruning (discussed above), which can be applied to many different types of markers, and considers a number of different models of demographic history, has been developed by Drummond et al (2002) (http://evolve.zoo.ox.ac.uk/beast/). This method allows for data to be taken at different times, enabling the use of ancient DNA samples.

For nuclear sequence data, the use of estimators such as Tajima's $D$ (Tajima, 1989) and Fu and Li's $D^{*}$ (Fu and Li, 1993) predominates. However, likelihood-based methods have also been used. For example, in a table of 12 published nuclear sequence data sets listed in Wall and Przeworski (2000), six publications used likelihoodbased analyses, in addition to inferences based on the estimators. Five of these used the method of Griffiths and Tavaré (1994), implemented in the Genetree program. Generally, in the studies that use Genetree, estimation of population growth is ancillary to the main goal of estimating $T_{\text {MRCA }}$. One publication uses the summarystatistic likelihood-based method of Fu and Li (1997) to estimate coalescence times. The Fluctuate program of Kuhner et al (1998) does not appear to have been used in studies of human population growth, but has been used extensively for other species (eg Griswold and Baker, 2002). Probably the widespread use of Genetree in human studies reflects the impact of the paper by Harding et al (1997), which was the first study that applied the program to a substantial data set. The growth model used in the published analyses with Genetree is of a population exponentially declining towards zero into the past: $N(t)=N_{0} \mathrm{e}^{-\beta t}$, where $N_{0}$ is the current population size and $\beta$ is the growth rate. Of the papers cited in Wall and Przeworski (2000), only those of Harding et al (1997) on beta-globin, and Thomson et al (2001) using Y chromosome sequence data provide information on the joint likelihood for $\theta$ (scaled mutation rate) and $\beta$. While the likelihood-based methods have clearly been important in making inferences about genealogical parameters from the sequenced data, it is not clear from these studies that their use for estimating population growth has provided greater insights than the results obtained with estimators such as Tajima's $D$, and it would appear that more recent papers have tended to use methods based on summary statistics (Pluzhnikov et al, 2002).

In microsatellites, most of the studies of population growth have been carried out with estimators designed to incorporate the microsatellite mutation mechanism (Kimmel et al, 1998; Reich and Goldstein, 1998). No likelihood-based method appears to have been used for the analysis of human population growth from autosomal microsatellite data, other than the example analysis in Beaumont (1999), which gave little evidence for population growth in a sample of Europeans surveyed at 10 tetranucleotide loci (data from Jorde et al, 1997). (For ease of description, 'nonautosomal' refers here to the nonrecombining $\mathrm{Y}$ chromosome and mtDNA, while 'autosomal' generally applies to recombining nuclear markers, referring to the X-chromosome explicitly where appropriate to avoid ambiguity.) Pritchard et al (1999) performed a Bayesian analysis on summary statistics from Y-chromosome microsatellites. The sample consisted of 445 chromosomes sampled from a number of different populations worldwide. They used a model where a population grows exponentially, with a growth rate, $r_{\mathrm{g}}$, from an ancestral population of fixed size, $N_{\mathrm{A}}$, at some time, $t_{a}$, generations ago. Using this method they were able to incorporate prior information about mutation rates, $\mu$, and demographic parameters, and were also able to investigate the effect of different models of microsatellite evolution. Marginal posterior distributions for $\mu, r_{\mathrm{g}}, N_{\mathrm{A}}$ and $t_{a}$ were then obtained. They estimated parameters in populations individually and in the pooled data, and the results strongly supported a model of population growth with estimates of the $T_{\text {MRCA }}$ around 50000 years (this figure is also supported by the estimates in Thomson et al, 2001), and estimates of the time since the onset of growth generally less than 20000 years, depending on the populations analysed, and the details of the mutation model. They were also able to make direct likelihood-ratio comparisons of a model with growth versus a model of stable population size, and strongly reject the latter possibility. This form of analysis illustrates the potential flexibility of Bayesian approaches, even if not all the information in the data is used.

A question of current interest that may be usefully addressed using likelihood is the discrepancy between the different types of markers in what they say about the timing and magnitude of past population growth (Brookfield, 2000; Harpending and Rogers, 2000; 
Przeworski et al, 2000; Wall and Przeworski, 2000; Rogers, 2001; Pluzhnikov et al, 2002). Essentially, nonautosomal markers show a very strong signal of population growth. Data from the Y-chromosome suggest a short time until the MRCA and a relatively recent population expansion, as described above (Pritchard et al, 1999; Thomson et al, 2001), and mtDNA markers suggest times of expansion of the order of 40000-100000 years using the sudden expansion model (Excoffier and Schneider, 1999). In contrast, autosomal data provide conflicting evidence of population growth, with patterns that vary among populations and loci studied (Wall and Przeworski, 2000). A simple way in which this could be addressed in a likelihood framework is to use hierarchical models where the parameters are allowed to vary among loci. Such an approach has been used for microsatellite markers (Storz and Beaumont, 2002; Storz et al, 2002). The idea is to try to detect discrepant loci that may be affected by selection. In principle, it would be possible to combine data from autosomal and nonautosomal analyses to try to detect incompatibilities, on the principle that even if special pleading is allowed (in terms of the differential behaviour of males and females), the demographic parameters for the autosomal markers need to be compatible with those of the nonautosomal markers.

A particular problem with likelihood-based approaches is that there is a wide variation in the demographic models assumed and in their parameterisation, which makes comparison between different studies rather difficult. The results in Figure 2 are an attempt to circumvent this partially by at least comparing the results of three separate studies on the same parameterisation. There are, however, still substantial

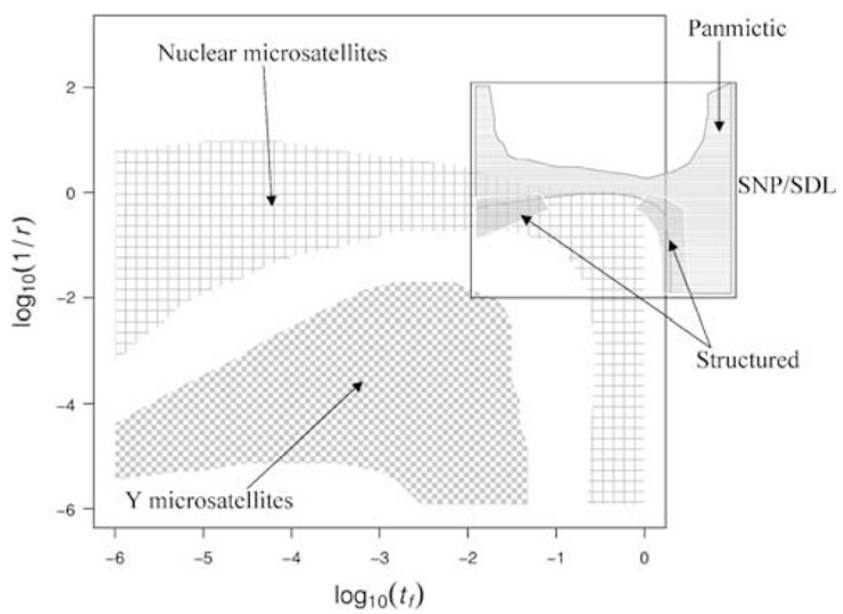

Figure 2 Confidence regions for demographic parameters from three separate analyses. The main figure shows the $90 \%$ highest probability density regions for 10 nuclear microsatellite markers, and for eight linked Y-chromosome microsatellites, as described in the text. The $y$-axis is the ancestral population size divided by the current population size on a $\log _{10}$ scale. The $x$-axis is the time in generations since the onset of growth divided by twice the current population size, also on a $\log _{10}$ scale. Inset shows the results from a combined analysis of SNP loci and the short stretch of sequence from which these were obtained (SDL, see text for details) taken from Figure 9a and b of Wakeley et al (2001). Two sets of confidence region are shown - either taking into account or ignoring population structure in the worldwide sample used for the analysis. differences in the details of the models and the provenance of the data sets. The autosomal microsatellite data set was analysed in Beaumont (1999), who gave a summary of the marginal posterior distributions for $r$ (the ratio of current to ancestral population size) and $t_{f}$ (time in generations since population changed in size, $t_{a}$ in the notation above, divided by the current population size, measured as number of chromosomes), assuming a model of linear population growth. The $90 \%$ highest probability density region (loosely, confidence region) estimated for the joint distribution on which these summaries were based is plotted in Figure 2. For comparison, the 90\% HPD region for the Y-chromosome data set analysed by Pritchard et al (1999) is also plotted using the same parameterisation. In this case, as described above, the posterior distribution is estimated from summary statistics, using a model of exponential population growth, and was re-estimated for this parameterisation using the method of Beaumont et al (2002). Finally, the 95\% confidence regions from Figure 9a and b in Wakeley et al (2001), based on an SNP data set from a worldwide sample of humans, described in more detail in the next section, are also included. The difference between the two sets of contours here is that in one case the effect of population structure is modelled, and in the other it is ignored. The model of population growth in this case is one of sudden expansion to the current size. It should be noted that the Wakeley et al (2001) analysis considers a slightly longer scaled time than the microsatellite analyses. As discussed in Beaumont (1999), this region of parameter space generally has a flat likelihood surface, and it is reasonable to assume that the confidence region for the nuclear microsatellites extends to overlap with that of the SNP data. Obviously, there are a number of additional caveats in the comparisons - the different provenance of the samples, the types of demographic model considered, and the methods of analysis - but it would appear that the Y-chromosome microsatellite data are not compatible with either the autosomal microsatellite data or the SNP data, whereas the two autosomal data sets, despite substantial differences in the types of markers and modelling assumptions, appear to be compatible, generally suggesting weak evidence of population expansion. With respect to the details of the expansion models, it should be noted that the results in Beaumont (1999) and Reich et al (1999) suggest that inferences about population growth are relatively insensitive to the details of how population size changes, provided that the changes are monotonic. A further observation is that if data from geographically separated populations are pooled, ignoring the effects of population structure, as in the Y-chromosome data here, any signal of population growth will tend to be weakened (Reich et al, 1999; Wakeley et al, 2001; Ptak and Przeworski, 2002), yet the observation of population growth is very strong in this data set. The observations in Beaumont (1999) suggest that the genealogies associated with the region of highest posterior density in the $\mathrm{Y}$ chromosome data set have long recent branches ('star genealogies'), whereas the genealogies associated with the region of highest posterior density in the other two data sets are more similar to those in stable populations.

There are a number of different explanations for the apparent discrepancy between the autosomal and non- 
autosomal markers. One possibility is that it simply arises from analysing the wrong demographic model. For example. Fay and Wu (1999) have suggested that an ancient bottleneck can account for the discrepancy. Since the size of nonautosomal genealogies is generally expected to be around $\frac{1}{4}$ of the autosomal genealogies, other things being equal, they may be unaffected by the bottleneck, because there is a higher chance that the time of the most recent common ancestor is more recent than the bottleneck. In contrast, the signal of population growth may be obscured in the longer genealogies of most autosomal loci by the presence of the bottleneck (see also Excoffier, 2002). Alternatively, genetic hitchhiking and recurrent selective sweeps or background selection against recurrent deleterious recessives may be prevalent and most strongly affect regions of low recombination, including the nonrecombining $\mathrm{Y}$ and mtDNA (Brookfield, 2000; Wall and Przeworski, 2000). Harpending and Rogers (2000) and Rogers (2001) have suggested that another possibility is that balancing selection is prevalent in the nuclear markers, obscuring the signal of population growth. If balancing selection were ubiquitous, it would predict that regions of low recombination in the nuclear marker data set would have longer genealogies, and therefore be more variable. Recent evidence has indicated a statistically significant positive correlation between levels of variability and local recombination rate (Nachman, 2001; Payseur and Nachman, 2002). This observation has been interpreted as favouring the selective sweep or background selection hypotheses, but has recently been suggested to arise from a correlation of mutation and recombination rates (Hellmann et al, 2003).

Analysis of population structure with gene-frequency data As with inference of population growth, estimation of patterns of migration has generally depended on method-of-moment estimators. Data have been visualised by ordination methods, such as principle component analysis or correspondence analysis based on gene frequencies. Other visualisation methods suitable for frequency data are metrical scaling, multidimensional scaling and the construction of neighbour-joining trees based on genetic distances. Sequence data are often converted into haplotype networks using various taxonomic methods. Good descriptions of these methods are given in (Excoffier, 2001; Rousset, 2001; Stumpf and Goldstein, 2001; Templeton, 2002; Hey and Machado, 2003). Traditionally, estimates of migration rates have been based on those of the average inbreeding coefficient within populations, $F_{\mathrm{ST}}$, although this practice has been criticised (Whitlock and McCauley, 1999). The problem is that differences in gene frequencies among subpopulation are used to estimate migration rates on the assumption that there is a balance between immigration, tending to homogenise gene frequencies and local inbreeding (drift), which tends to cause them to differ among populations. However, the assumption of equilibrium need not apply and therefore estimates of gene flow may be false. Clearly, if this is regarded as the main caveat to the estimation of migration rates then likelihood estimates from models that also assume equilibrium will suffer from the same drawback, even if, given the model assumptions, the estimates of gene flow are more precise.

There are a number of likelihood-based methods for estimating migration rates between different populations (see Rousset, 2001, for a review). Two of these methods estimate parameters in the standard structured coalescent model (Nordborg, 2001), whereas the others make simplifying assumptions about population structure. The method of Bahlo and Griffiths (2000) uses the importance sampling approach of Griffiths and Tavare (1994), and is implemented in the program Genetree. Beerli and Felsenstein $(1999$, 2001) have developed a method, implemented in the computer program Migrate (http:// evolution.genetics.washington.edu/lamarc.html), based on the importance sampling approach in Kuhner et al (1995). Unlike earlier estimators of gene flow, these methods allow for immigration rates from each population into each recipient population to be estimated separately. Beerli and Felsenstein (2001) apply their method to mtDNA sequence samples from Nile populations of humans from Egypt, Nubia and Sudan. They are able to demonstrate substantial gene flow both from Egypt and Sudan into Nubia, of the order of $N_{\mathrm{f}} m=10-15$, where $N_{\mathrm{f}}$ is the number of females in the population and $m$ is the immigration rate. Immigration into Egypt and Sudan is substantially less (around $N_{\mathrm{f}} m=1.5-2.5$ ). No other publications thus far appear to have used the Migrate software for the analysis of gene flow in human populations. One publication has used Genetree in this context (Bosch et al, 2001). In this study, a number of samples of Y-chromosomes from populations in North African and the Iberian Peninsula were genotyped using a mixture of biallelic (SNPs and indels) and microsatellite markers. The bulk of the analysis was based on a description of the detailed haplotype networks that could be obtained by standard taxonomic methods. However, Genetree was used to estimate migration between the two regions, and to estimate times of occurrence of key mutations in the genealogy. Inferences for these parameters were conditional on the maximumlikelihood estimates of scaled mutation rate and population growth rate. Estimates were also made assuming no population growth. Using likelihood ratio tests, they were able to reject a model of panmixia, and estimated migration rates of $N_{\mathrm{m}} m=1.25$ from Iberia to NW Africa and $N_{\mathrm{m}} m=2$ from NW Africa to Iberia, where $N_{\mathrm{m}}$ is the number of males in the population and $m$ is the immigration rate. The times estimated for several mutations were used in conjunction with the haplotype networks to make additional inferences about demographic history.

A simplified model of population structure is the island model (Wright, 1931) where immigrants have equal probability of coming from any of the subpopulations. In the limit of a large number of islands and low mutation rate, the probability distribution of gene frequencies among populations is given by a Dirichlet distribution (Wright, 1951). The likelihood for finite samples taken from this distribution is given by the multinomial-Dirichlet distribution (Balding and Nichols, 1994, 1995, 1997; Rannala and Hartigan, 1996; Holsinger, 1999; Holsinger et al, 2002). The genealogy for a subpopulation is illustrated in Figure 1f. Whenever an immigration event occurs in the genealogy, the allelic type is sampled from the baseline frequency. These 
methods, unlike the others above, can only be used to estimate the total immigration rates into each population (or, equivalently, the inbreeding coefficients), rather than identify immigration from each individual population separately. However, given the strong assumptions in the former methods that gene flow is only between the populations used in the study, this latter more modest approach may be more robust. Another useful feature of the method of Balding and Nichols (1997) implemented in the program FSTMET (http://www.reading.ac.uk/ Statistics/genetics/software/fstmet/) is that it allows a hierarchical approach to modelling where immigration rates are allowed to vary among loci (see also Balding 2003). As discussed above, in relation to models of demographic growth, such an approach is useful to identify loci that might be under selection. Moreover, in structured populations, there is the potential for divergent selection pressures among populations due to local adaptation, which makes it more likely that discrepant loci can be identified, as originally appreciated by Lewontin and Krakauer (1973). For more recent moment-based approaches to the problem, see Beaumont and Nichols (1996), Vitalis et al (2001), and Schlötterer (2002). Again, as with the method of Rannala and Hartigan (1996), other than in studies by the authors (primarily in a forensic setting), there appear to have been no applications of the methodology of Balding and Nichols (1997) in human population genetics.

Recently, Wakeley et al (2001) have used the subdivided-population model of Wakeley $(1999,2001)$ to analyse SNP data from a number of human populations. The data have been used to infer immigration rates, past population growth and the effects of ascertainment bias. The raw data consist of short segments ( $\sim 500 \mathrm{bp})$ of DNA, which the authors term SNP-discovered loci (SDLs), chosen to have at least two segregating sites in the samples from which the SNPs are identified. Given the complexity of the data and computational constraints, the analysis necessarily consists of a mixture of moment-matching and likelihood-based analysis. First, the immigration rates and scaled mutation rates are estimated jointly using moment matching, assuming a model of no growth. Then, conditioning on the estimates of immigration, the joint likelihood is estimated for the ratio of ancestral to current size, time since the population changed in size in units of current size, and the mutation rate (scaled by current size). Profile likelihood surfaces for the two demographic parameters are presented, where likelihoods are estimated by conditioning on the maximum-likelihood estimate of theta for each combination of the demographic parameters. An overall analysis is also carried out using both the number of SNPs per SDL (ie the number of segregating sites) and the frequencies of the SNPs. The authors point out that the process of ascertainment of SNPs will tend to lead to SDLs with a lower variance in the number of SNPs per SDL, supporting a model of population growth, and intermediate frequencies for each SNP, supporting a model of decline. Thus, the process of ascertainment differs in its effects depending on the genetic information used. In their analysis ascertainment bias leads to these theoretically expected effects, and they suggest that ascertainment bias may explain the finding of population decline in Wakeley (1999). They also find a strong ascertainment effect on the estimation of immigration rates, which tend to be substantially overestimated if ascertainment is ignored. In addition, ignoring population structure tends to obscure the signal of population growth in the data, as discussed in the previous section.

These methods all assume equilibrium between gene flow and drift. Nielsen and Slatkin (2000) and Nielsen and Wakeley (2001) consider methods for estimating parameters in a model of two current populations that have diverged from a common ancestral population at time $t$ in the past. After divergence there is gene flow at a constant rate. The parameters that can be estimated are the time since divergence, scaled by the size of one of the current populations $T=t / 2 N_{1}$, the ratio of the current population sizes $r=N_{2} / N_{1}$, the ratio of the ancestral size to the size of one of the current populations $a=N_{\mathrm{A}} / N_{1}$ and the scaled immigration rates, $M_{1}=2 N_{1} m_{1}$ and $M_{2}=2 N_{2} m_{2}$. The method implemented in Nielsen and Wakeley uses MCMC to obtain marginal posterior distributions (or 'integrated likelihoods', since flat priors are assumed) for the parameters of interest. Using simulations, they were able to show that the method has good power to distinguish the possibilities of migration versus no migration between the two populations, and the use of likelihood appeared to give substantially greater power than an earlier momentbased method (Wakeley, 1996). They apply the method to mtDNA data from two populations of sticklebacks, and are able to show that the data are compatible with a long divergence time and migration between them. With this data set, they are also able to detect unequal migration rates between the two populations, and unequal sizes of the two current populations. Clearly, the method has wide applicability, and although recently used in a study of chaffinches (Griswold and Baker, 2002), there are no published applications that use it for human data.

Without migration, the model of Nielsen and Wakeley becomes similar to that considered by Nielsen (1998), where dating the divergence time of two populations is the main focus. This can be generalised to many populations that split in a branching manner. For linked microsatellite loci the program Batwing (http:// www.maths/abdn.ac.uk/ ijw), based on the model in Wilson and Balding (1998) and Wilson et al (2003), can be used to obtain posterior distributions of population trees in a manner similar to those obtained in taxonomic analysis. Likelihood-based analyses of population splitting based on gene frequencies have been carried out using the assumption that there have been no mutations since the time of splitting, and frequencies differ among populations due to genetic drift. These drift-based models have a long history, exemplified by the CONTML package in Phylip (Felsenstein, 1981), which uses the approximate method of Cavalli-Sforza and Edwards (1967) for inferring population trees.

Drift-based models are well suited to addressing conservation problems because of the recent and widespread effects of habitat fragmentation, and the general interest in how recent inbreeding can increase the risk of population extinction (reviewed in Beaumont, 2001). For short time scales, it is possible to compare the likelihood of a model of divergence through drift with the likelihood under the infinite island model with a low mutation rate, given by the multinomial-Dirichlet distribution discussed above (Beaumont, 2001), thereby addressing problems similar to those in Nielsen and 
Wakeley (2001), but with multiple populations over a recent timescale. This approach has been used in a conservation management setting (Ciofi et al, 1999; Goodman et al, 2001), but does not appear to have been used for human data. Indeed, there has been relatively little interest in the use of nonmutation models for human population genetics. However, the availability of SNP gene-frequency data has kindled interest in this approach because their mutation rate is sufficiently low so that it is reasonable to assume that most of the variability occurred before the creation of recent human population structure through processes such as colonisation, settlement, extinction, admixture and immigration.

A recent example of the use of this modelling approach in human population genetics is Nicholson et al (2002), who analyse SNP gene frequency data sampled from a number of populations. The general idea is that the SNP sample is a random draw from some unknown subpopulation gene frequency, which is in turn a random draw from a distribution with the mean corresponding to the baseline gene frequency, $p$, (shared between populations) and variance $c p(1-p)$. Although considered as a statistical parameter, $c$ approximates $T / N_{\mathrm{e}}$ in the standard Wright-Fisher diffusion model, where $T$ is the time of the split and $N_{\mathrm{e}}$ is the harmonic mean effective size over the interval. This is similar to the model of Cavalli-Sforza and Edwards (1967), who approximated drift as Brownian motion on an arcsinetransformed scale. Using MCMC, Nicholson et al (2002) are able to integrate out the unknown gene frequencies and obtain marginal posterior distributions for individual $c_{j}$ for the $j$ th population. Nicholson et al's model ascertainments by assuming a large number of potential loci are examined in (generally small) samples from each of a number of subpopulations, and a locus is chosen if it is not fixed for the same allele in all subpopulations. The likelihood is then multiplied by the probability of ascertaining the loci, given the gene frequencies in the subpopulations. They find that the ascertainment effect on estimates of the $c_{j}$ is small, which contrasts with the finding of Wakeley et al (2001), who found ascertainment effects both in the estimation of immigration rates within populations and in estimates of population growth. It is not clear what the cause of this discrepancy is, but there are differences in the ascertainment model used, the model of population structure and the method of estimation. Nicholson et al (2002) show that their approach generally outperforms moment-based estimators of $F_{\mathrm{ST}}$, and they apply it to data from a number of populations. They show that there is very little differentiation among the European populations they studied (83 loci; France, Utah, Iceland; Wang et al, 1998), although some support for further divergence in the Iceland sample. There was considerably more divergence in another data set based on 66 loci surveyed in a population of pygmies, a Melanesian population, a mixed European and a mixed Chinese population (Bowcock et al, 1991a,b), with estimates of $c$ in the pygmies and the Melanesian population of around 0.4, suggesting a substantial drift in comparison with the mixed European sample, which had $c=0.05$. Using the model, it is also possible to examine the residuals for goodness-of-fit. In particular, one can test for correlations among the residuals for different subpopulations to detect shared recent ancestry of populations.
A model that is related to the drift-based model considered above is that of admixture followed by drift (Figure 1d). This type of model has a long history (reviewed in Beaumont, 2001). In the model of Chikhi et al (2001), a hybrid population is formed at some time $T$ in the past in which the initial gene frequencies are sampled in proportion, $P_{\text {mix }}$, from one parental type, and $\left(1-P_{\text {mix }}\right)$ from the other. The two parental populations and the hybrid population are then isolated and their gene frequencies change over the time interval $T$ due to drift. The method then uses MCMC and importance sampling to obtain posterior distributions for $T / N_{\mathrm{e}}$ in each of the three populations and $P_{\text {mix }}$. This model is very similar to the formulation of the Icelandic admixture problem by Thompson (1973). By using MCMC, unlike previous approaches, marginal or joint posterior distributions (in fact, proportional to the marginal likelihoods, because uniform priors were used) for all four parameters can be obtained. More recently, Wang (2003), extending a method for modelling drift described in Wang (2001), has addressed the problem of inferring admixture using likelihood. This approach is particularly interesting in that, by clever numerical approximations that appear to work well for drift-based problems, it avoids the use of Monte Carlo sampling methods, and is substantially faster than that of Chikhi et al (2001). The method allows profile likelihood surfaces to be calculated for the parameters of interest. The admixture model is similar to that in Chikhi et al (2001) but also includes parameters for the degree of divergence of the parental populations. Wang (2003) shows that modelling the degree of divergence of the parental populations is important for good estimation, and that the assumption of independent uniform priors for the gene frequencies in the parental populations, as in Chikhi et al (2001), can lead to poor inference of the admixture proportions. Wang (2003) demonstrates, using simulations, that the likelihood method is more accurate than moment-based methods, and illustrates its use on a data set of nuclear markers (primarily RFLP) surveyed in African-American populations by Parra et al (1998).

\section{Analysis of population structure with genotypic data}

Recently, with the prevalence of many different genetic markers that can be typed within a single individual, there has been interest in using multilocus genotypic information to identify recent immigrants and hybrids and assign them to candidate populations, and to identify cryptic population structure (see Beaumont, 2001, for a review of the history of this approach). In human population genetics, this approach is useful in association mapping because spurious linkage disequilibrium caused by cryptic population structure can be identified (Pritchard et al, 2000b). The method of Pritchard et al (2000a), and an application that uses it, is described below (see also recent studies by Dawson and Belkhir, 2001; Anderson and Thompson, 2002; Wilson and Rannala, 2003; Falush et al, 2003).

Pritchard et al (2000a) consider two scenarios. The simplest case is the no-admixture model (Figure 1g), where the sample is assumed to consist of a mixture of individuals from each of two populations that have different gene frequencies. If the parental populations differ in frequency and if individuals come from one or 
other, then there will be departures from HardyWeinberg (Wahlund effect) and gametic phase equilibrium in the sample considered as a whole because the genotypes of individuals have a low probability of arising from random sampling of the sample gene frequencies. The aim of the analysis, given the putative number of parental populations, is to identify the distribution of gene frequencies within them, and the probable provenance of individuals, consistent with the data. Thus, in this case, there are two populations with frequencies $\mathbf{x}_{1}$ and $\mathbf{x}_{2}$. For each individual, with genotype $G_{i}$, there is an indicator variable, $z_{i}$, that takes a value (eg 1,2) specifying where it comes from. The alleles at each locus in each individual are assumed to be independently and randomly drawn from the frequencies $\mathbf{x}_{1}$ or $\mathbf{x}_{2}$. The alleles are drawn from either $\mathbf{x}_{1}$ or $\mathbf{x}_{2}$ depending on whether $z_{i}$ is 1 or 2 for the $i$ th individual. The likelihood for the set of genotypes, $G$, is then $p\left(G \mid \mathbf{x}_{1}, \mathbf{x}_{2}, \mathbf{z}\right)$, where the probabilities are multiplied across loci and individuals. MCMC is then used to obtain the posterior distribution $p\left(\mathbf{x}_{1}, \mathbf{x}_{2}, \mathbf{z} \mid G\right)$. The mean $p\left(z_{i}=1\right)$ over the MCMC run then gives the probability that individual $i$ comes from population 1, marginal to any particular value of $\mathbf{x}_{1}, \mathbf{x}_{2}$.

In the admixture scenario, each of the two alleles at each locus in an individual can be randomly and independently drawn from either population. Thus for the $j$ th allele (where $j$ takes the value either 1 or 2 ) in the $i$ th individual, the indicator variable $z_{i j}$ specifies which of the two populations the $j$ th allele comes from. Also, the proportion of the genotype that comes from population $1, q_{i}$, is specified for each individual. The likelihood is then $p\left(G \mid \mathbf{x}_{1}, \mathbf{x}_{2}, \mathbf{q}, \mathbf{z}\right)$. Using MCMC the posterior distribution $p\left(\mathbf{x}_{1}, \mathbf{x}_{2}, \mathbf{q}, \mathbf{z} \mid G\right)$ is obtained, and, generally the interest is in reporting posterior means and credible intervals for $q_{i}$, marginal to the other parameters.

Although the above description is for two populations, in general, $K$ populations can be specified. From the MCMC simulation, the marginal likelihood $p(G \mid K)$ can be estimated (ie independent of $\mathbf{x}, \mathbf{z}$ and $\mathbf{q}$ ). By varying $K$ among different runs, it is then possible to estimate the posterior probability $p(K \mid G)$, to infer the number of populations contributing to the sample, independent of any particular estimate of the parental gene frequencies, provenance of individual segments of chromosome or degree of admixture of individuals.

An assumption of the approach is that the individuals, and associated $z_{i}$ or $z_{i j}$, are all sampled independently, and that within individuals, conditional on the $z_{i}$ (or $z_{i j}$ ), the segments of chromosome are all sampled independently. Obviously linkage disequilibrium in the parental populations will violate this, but even if the parental populations are in equilibrium, the sampling of linked markers may violate this assumption unless the admixture is ancient. For example, under the admixture model, if admixture is recent, the indicator variables $z_{i j}$ should be assumed to have the same value in the $i$ th individual for all $j$ segments that are tightly linked on the same chromosome. Ideally, the linkage information from tightly linked markers should be useful for inferring time scales and degrees of admixture; however, an extension of the modelling approach would probably also need to consider genealogical structure in addition to recombination.
An example application to human population genetics is the study by Wilson et al (2001), who use the model of Pritchard et al (2000a) to identify population structure in a worldwide sample of humans taken from eight regions, and its association with polymorphism in drug-metabolising enzymes (DMEs). Wilson et al (2001) use a sample typed for 16 chromosome, one microsatellites and $23 \mathrm{X}$ linked microsatellites. The minimum map distance between any pair of markers was $2 \mathrm{cM}$. Using the admixture model, they inferred four populations corresponding geographically to Western Eurasia, Sub-Saharan Africa, China and New Guinea. Subsets of loci, or all the loci from a particular chromosome, tended to support slightly different groupings. An average membership of the four groups could be estimated for the eight regions by averaging the $q_{i}$ across individuals within each region. In general, average membership for each region was predominantly to a single genetic group rather than split across groups, although the group membership of some regions (for example Afro-Caribbean and Ethiopia) suggested a fair degree of admixture. Wilson et al (2001) found strong differences in allele frequencies of the DMEs among the four inferred groupings, and were able to show that if traditional groupings of the populations were used (Caucasian, Black, Asian), the gene-frequency differences were strongly attenuated. This study emphasises the need to model demographic history correctly when making inferences about genetic markers that are subject to selection.

A larger scale study has been reported by Rosenberg et al (2002), who have applied the method of Pritchard et al (2000a) to a large worldwide sample scored for 377 microsatellite loci. They inferred six groups from these data, corresponding to five large geographic regions Africa, Eurasia, East Asia, Oceania and America. Native Americans were not included in the study of Wilson et al (2001). Within each of the main groups further finer geographically related groupings could be found. In contrast to the study of Wilson et al (2001), Rosenberg et al (2002) conclude that self-reported ancestry may often be adequate for the assessment of, for example, individual disease risk.

Another example of the use of genotypic models to study human population structure is that of Overall and Nichols (2001), who have developed a method for distinguishing whether departures from HardyWeinberg equilibrium leading to homozygote excesses are due to cryptic population structure, or due to consanguineous mating. The essential intuition that allows these two possibilities to be distinguished is that with a cryptic population structure, provided all subpopulations are equally inbred, measured by the parameter $\theta$, homozygosity should be uncorrelated among loci within individuals - that is, knowing that an individual is homozygous at one locus says nothing about other loci. In contrast, consanguineous mating will result in offspring that have a higher probability of homozygosity at each locus. Obviously, correlations can also arise if there is a cryptic population structure, but the inbreeding coefficient varies among subpopulations. The probability of obtaining the multilocus genotype of each individual can be calculated as a function of the proportion of individuals that arise from a consanguineous mating, $C$, and the inbreeding coefficient $\theta$, and this can be multiplied over all individuals, giving the 
likelihood $p(G \mid C, \theta)$. Overall and Nichols (2001) estimated likelihood surfaces for samples taken from two Asian communities in the UK, in which prior genetic analysis had indicated statistically significant homozygote excess. The sample sizes were approaching 100 individuals from each community, typed for six to 10 loci. Consanguinity was modelled as marriage between first cousins. In one population, the Mirpuri, it was known a priori that $50 \%$ of the individuals were from marriages between first cousins. The other population, the Jullunduri, had no tradition of consanguineous marriage, and there was interest as to what was the cause of the homozygote excess. For the Mirpuri sample, the maximum-likelihood estimate indicated 50\% first cousin marriage, and a low value of $\theta$. The Jullunduri sample had two modes, suggesting either $100 \%$ consanguinity and small $\theta$, or no consanguinity and $\theta \sim 0.06$. Unsurprisingly, given the relatively low number of markers available, the likelihood surfaces for both data sets were quite broad, and consistent with most values of $C$, and values of $\theta<0.1$. However, the method clearly is of potential value in elucidating the finer details of human population structure.

Methods based on multilocus genotypes are currently being rapidly developed in many directions. The use of anonymous markers, which can generally be assumed to have a recombination rate of 0.5 , allows direct calibration of genealogical processes, and hence enables demographic parameters such as immigration and effective population size to be estimated directly, rather than scaled in terms of each other. An example of this use is the moment-based method of Vitalis and Couvet (2001), which allows for separate identification of effective population size and immigration rate into populations. Related developments, possibly of less use in human population genetics but of great utility elsewhere, are methods that use multilocus information to infer the number of sibships in a sample (Smith et al, 2001) and the number of parents in a sample (Emery et al, 2001).

\section{Conclusions}

The purpose of this article has been to highlight recent approaches to genetic data analysis, and their potential application in human population genetics. These methods allow full use of information in the data. Even when not all the information is used, as in those that estimate the likelihood or posterior distribution from summary statistics, there are still many advantages over standard moment-based methods. For example, it is possible to incorporate background information into the model, infer parameters directly rather than scaled in terms of each other, infer parameters jointly, infer parameters without conditioning on particular values of other parameters and visualise the distribution of most probable parameters given the data, which facilitates exploratory data analysis.

The application of these methods to human data appears to have been somewhat patchy, and standard moment-based methods are still predominantly used. In general, it would appear that likelihood-based methods have been much more rapidly taken up in nonhuman population genetics. This may point to an inherent conservatism in the subject area, and also probably reflects the fact that while humans are the most intensively studied organism, the great majority of population genetic studies are on nonhumans. An obvious problem with the use of sampling-based approaches is the length of time required to get an answer. A criticism of Bayesian inference in population genetics is the sensitivity of the results to the assumed priors, particularly when making inferences about nonidentifiable parameters. This criticism can be countered by exploring the effects of different priors, but has hitherto been difficult because of the time taken to run the simulations. Probably, a 100-fold increase in computational speed is needed to allow flexible, exploratory, repeated use of these methods on most standard data sets. The parallelism inherent in most sampling methods will undoubtedly benefit from the trend towards massively parallel cluster-based computing. With increasing use of software libraries, such as MPICH, to spawn independent sampling calculations onto many processors in a cluster, a 100-fold speed-up of most methods should be immediately practicable.

Although it is difficult to predict the future direction of these methods, it seems clear that likelihood-based inference with summary statistics will be increasingly used in future. It lends itself particularly well to parallel computation, and is applicable to many population genetics models, not necessarily based on the coalescent. For example, it has been used by Tishkoff et al (2001) to infer patterns of selection at the G6PD locus in humans. The summary-statistic method may be a particularly fruitful first step when information from linkage disequilibrium in recombining markers is used to infer demographic history (Wall, 2000). The potential additional genealogical information that may arise, particularly when SNP data are used, makes likelihood-based inference with recombining markers particularly attractive. It is difficult to envisage how the summary-statistic method can be used in multilocus genotypic analyses, where the number of summary statistics and parameters is necessarily of an order similar to the number of individuals. Methods that estimate the full-data likelihood for recombining markers have recently been developed (Kuhner et al, 2000; Nielsen, 2000; Fearnhead and Donnelly, 2001), and it is possible to envisage that extensions of the coalescent with recombination (Nordborg, 2000) will eventually merge with multilocus genotypic methods, which currently do not incorporate genealogical processes.

\section{Acknowledgements}

I am grateful to Molly Przeworski and John Wakeley for a useful correspondence, and for comments on previous versions of the paper, and to Lounes Chikhi for comments and for providing references and access to unpublished material. While writing this review, the author was supported initially by the Research Endowment Trust Fund of the University of Reading, and more recently by a Natural Environment Research Council Advanced Fellowship.

\section{References}

Anderson EC, Thompson EA (2002). A model-based method for identifying species hybrids using multilocus genetic data. Genetics 160: 1217-1229. 
Anderson EC, Williamson EG, Thompson EA (2000). Monte Carlo evaluation of the likelihood for $N_{e}$ from temporally spaced samples. Genetics 156: 2109-2118.

Bahlo M, Griffiths RC (2000). Inference from gene trees in a subdivided population. Theor Popul Biol 57: 79-95.

Balding DJ (2003). Likelihood-based inference for genetic correlation coefficients. Theor Popul Biol 63: 221-230.

Balding DJ, Nichols RA (1994). DNA profile match probability calculation: how to allow for population stratification, relatedness, database selection and single bands. Forensic Sci Int 64: 125-140.

Balding DJ, Nichols RA (1995). A method for quantifying differentiation between populations at multi-allelic loci and its implications for investigating identity and paternity. Genetica 96: 3-12

Balding DJ, Nichols RA (1997). Significant genetic correlations among Caucasians at forensic DNA loci. Heredity 78: 583-589.

Beaumont MA (1999). Detecting population expansion and decline using microsatellites. Genetics 153: 2013-2029.

Beaumont MA (2001). Conservation genetics. In: Balding DJ, Bishop M, Cannings C (eds) The Handbook of Statistical Genetics. John Wiley: New York, pp 779-812.

Beaumont MA (2003). Estimation of population growth or decline in genetically monitored populations. Genetics 164: $1139-1160$.

Beaumont MA, Nichols RA (1996). Evaluating loci for use in the genetic analysis of population structure. Proc $R$ Soc Lond Ser B 263: 1619-1626.

Beaumont MA, Zhang W, Balding DJ (2002). Approximate Bayesian computation in population genetics. Genetics 162 2025-2035.

Beerli P, Felsenstein J (1999). Maximum-likelihood estimation of migration rates and effective population numbers in two populations using a coalescent approach. Genetics 152: 763-773

Beerli P, Felsenstein J (2001). Maximum likelihood estimation of a migration matrix and effective population sizes in $n$ subpopulations by using a coalescent approach. Proc Natl Acad Sci USA 98: 4563-4568.

Bosch E, Calafell F, Comas D, Oefner PJ, Underhill PA, Bertranpetit J (2001). High-resolution analysis of human Y-chromosome variation shows a sharp discontinuity and limited gene flow between Northwestern Africa and the Iberian Peninsula. Am J Hum Genet 68: 1019-1029.

Bowcock A, Hebert J, Mountain J, Kidd J, Rogers J, Kidd K et al (1991a). Study of an additional 58 DNA markers in ve populations from four continents. Gene Geogr 5: 151-173.

Bowcock A, Kidd J, Mountain J, Hebert J, Carotenuto L, Kidd K et al (1991b). Drift, admixture, and selection in human evolution: a study with DNA polymorphisms. Proc Natl Acad Sci USA 88: 839-843.

Brookfield JFY (2000). Human evolution: how recent were the $Y$ chromosome ancestors? Curr Biol 10: R722-R723.

Cann RL, Stoneking M, Wilson AC (1987). Mitochondrial DNA and human evolution. Nature 325: 31-36.

Cavalli-Sforza LL, Edwards AWF (1967). Phylogenetic analysis: models and estimation procedures. Evolution 32: 550-570.

Cavalli-Sforza LL, Feldman MW (2003). The application of molecular genetic approaches to the study of human evolution. Nat Genet 33: 266-275.

Chen Y, Liu JS (2000). In: Wilson L, Stephens DA, Harding RM et al. Inference in molecular population genetics - Discussion. J Roy Stat Soc Ser B 62: 636-655.

Chikhi L, Bruford MW, Beaumont MA (2001). Estimation of admixture proportions: a likelihood-based approach using Markov chain Monte Carlo. Genetics 158: 1347-1362.

Ciofi C, Beaumont MA, Swingland IR, Bruford MW (1999). Genetic divergence and units for conservation in the Komodo dragon Varanus komodoensis. Proc R Soc Lond Ser B 266: $2269-2274$
Clark AG, Nielsen R, Signorovitch J, Matise TC, Glanowski S, Heil J et al (2003). Linkage disequilibrium and inference of ancestral recombination in 538 single-nucleotide polymorphism clusters across the human genome. Am J Hum Genet 73: 285-300.

Dawson KJ, Belkhir K (2001). A Bayesian approach to the identification of panmictic populations and the assignment of individuals. Genet Res 78: 59-77.

Donnelly P, Tavaré S (1995). Coalescents and genealogical structure under neutrality. Annu Rev Genet 29: 410-421.

Drummond AJ, Nicholls GK, Rodrigo AG, Solomon W (2002). Estimating mutation parameters, population history and genealogy simultaneously from temporally spaced sequence data. Genetics 161: 1307-1320.

Emery AM, Wilson IJ, Craig S, Boyle PR, Noble LR (2001). Assignment of paternity groups without access to parental genotypes: multiple mating and developmental plasticity in squid. Mol Ecol 10: 1265-1278.

Estoup A, Clegg SM (2003). Bayesian inferences on the recent island colonization history by the bird Zosterops lateralis lateralis. Mol Ecol 12: 657-674.

Estoup A, Wilson IJ, Sullivan C, Cornuet J-M, Moritz C (2001). Inferring population history from microsatellite and enzyme data in serially introduced cane toads, Bufo marinus. Genetics 159: 1671-1687.

Excoffier L (2001). Analysis of population subdivision. In: Balding DJ, Bishop M, Cannings C (eds) Handbook of Statistical Genetics. Wiley: New York, pp 271-307.

Excoffier L (2002). Human demographic history: refining the recent African origin model. Curr Opin Genet Dev 12: 675-682.

Excoffier L, Schneider S (1999). Why hunter-gatherer populations do not show signs of Pleistocene demographic expansions. Proc Natl Acad Sci USA 96: 10597-10602.

Falush D, Stephens M, Pritchard JK (2003). Inference of population structure using multilocus genotype data: linked loci and correlated allele frequencies. Genetics 164: 1567-1587.

Fay JC, Wu C-I (1999). A human population bottleneck can account for the discordance between patterns of mitochondrial versus nuclear DNA variation. Mol Biol Evol 16: 1003-1005.

Fearnhead P, Donnelly P (2001). Estimating recombination rates from population genetic data. Genetics 159: 1299-1318.

Felsenstein J (1981). Evolutionary trees from gene frequencies and quantitative characters: finding maximum likelihood estimates. Evolution 35: 1229-1242.

Felsenstein J (1992). Estimating effective population size from samples of sequences: inefficiency of pairwise and segregating sites as compared to phylogenetic estimates. Genet Res 59: 139-147.

Felsenstein J, Kuhner MK, Yamato J, Beerli P (1999). Likelihoods on coalescents: a Monte Carlo sampling approach to inferring parameters from population samples of molecular data. In: Seillier-Moiseiwitsch F (ed) Statistics in Molecular Biology. IMS Lecture Notes - Monograph Series, Vol. 33. Institute of Mathematical Statistics and American Mathematical Society: Hayward, CA, pp 163-185.

$\mathrm{Fu} \mathrm{Y}$-X, Li W-H (1993). Statistical tests of neutrality of mutations. Genetics 133: 693-709.

$\mathrm{Fu} \mathrm{Y}$-X, Li W-H (1997). Estimating the age of the common ancestor of a sample of DNA sequences. Mol Biol Evol 14: 195-199.

Goldstein DB, Chikhi L (2002). Human migrations and population structure: what we know and why it matters. Annu Rev Genom Hum Genet 3: 129-152.

Goodman SJ, Tamate HB, Wilson R, Nagata J, Tatsuzawa S, Swanson GM et al (2001). Bottlenecks, drift and differentiation: the population structure and demographic history of sika deer (Cervus nippon) in the Japanese archipelago. Mol Ecol 10: 1357-1370.

Griffiths RC, Tavaré S (1994). Simulating probability distributions in the coalescent. Theor Popul Biol 46: 131-159. 
Griswold CK, Baker AJ (2002). Time to the most recent common ancestor and divergence times of populations of common chaffinches (Fringilla coelebs) in Europe and North Africa: insights into pleistocene refugia and current levels of migration. Evolution 56: 143-153.

Hey J, Machado CA (2003). The study of structured populations - new hope for a difficult and divided science. Nat Rev Genet 4: 535-543.

Harding RM, Fullerton SM, Griffiths RC, Bond J, Cox JM, Schneider JA et al (1997). Archaic African and Asian lineages in the genetic history of modern humans. Am J Hum Genet $\mathbf{6 0}$ 772-789.

Harpending H, Rogers A (2000). Genetic perspectives on human origins and differentiation. Annu Rev Genom Hum Genet 1: 361-385.

Hellmann I, Ebersberger I, Ptak SE, Pääbo S, Przeworski M (2003). A neutral explanation for the correlation of diversity with recombination rates in humans. Am J Hum Genet $\mathbf{7 2}$ 1527-1535.

Holsinger KE (1999). Analysis of genetic diversity in geographically structured populations: a Bayesian perspective. Hereditas 130: 245-255.

Holsinger KE, Lewis PO, Dey DK (2002). A Bayesian approach to inferring population structure from dominant markers Mol Ecol 11: 1157-1164.

Hudson RR (1991). Gene genealogies and the coalescent process. In: Futuyama KJ, Antonovics J (eds) Oxford Surveys in Evolutionary Biology. Oxford University Press, Oxford, pp $1-44$.

Jorde LB, Rogers AR, Bamshad M, Watkins WS, Krakowiak PA, Sung $S$ et al (1997). Microsatellite diversity and the demographic history of modern humans. Proc Natl Acad Sci USA 94: 3100-3103.

Kimmel M, Chakraborty R, King JP, Bamshad M, Watkins WS, Jorde LB (1998). Signatures of population expansion in microsatellite repeat data. Genetics 148: 1921-1930.

Kuhner MK, Yamato J, Felsenstein J (1995). Estimating effective population size and mutation rate from sequence data using Metropolis-Hastings sampling. Genetics 140: 1421-1430.

Kuhner MK, Yamato J, Felsenstein J (1998). Maximum likelihood estimation of population growth rates based on the coalescent. Genetics 149: 429-434.

Kuhner MK, Yamato J, Felsenstein J (2000). Maximum likelihood estimation of recombination rates from population data. Genetics 156: 1393-1401.

Lewontin RC, Krakauer J (1973). Distribution of gene frequency as a test of the theory of selective neutrality of polymorphisms. Genetics 74: 175-195.

Marjoram P, Molitor J, Plagnol V, Tavaré S (2003). Markov chain Monte Carlo without likelihoods. Proc Natl Acad Sci USA 100 15324-15328.

Markovtsova L, Marjoram P, Tavaré S (2000). The effects of rate variation on ancestral inference in the coalescent. Genetics 156: 1427-1436.

Nachman MW (2001). Single nucleotide polymorphisms and recombination rate in humans. Trends Genet 17: 481-485.

Nicholson G, Smith AV, Jonsson F, Gustaffson O, Stefansson K, Donnelly P (2002). Assessing population differentiation and isolation from single nucleotide polymorphism data. $J R$ Stat Soc Ser B 64: 695-715.

Nielsen R (1998). Maximum likelihood estimation of population divergence times and population phylogenies under the infinite sites model. Theor Popul Biol 53: 143-151.

Nielsen R (2000). Estimation of population parameters and recombination rates from single nucleotide polymorphisms. Genetics 154: 931-942.

Nielsen R, Slatkin M (2000). Analysis of population subdivision using di-allelic models. Evolution 54: 44-50.

Nielsen R, Wakeley J (2001). Distinguishing migration from isolation: a Markov chain Monte Carlo approach. Genetics 158: $885-896$.
Nielsen R, Mountain JL, Huelsenbeck JP, Slatkin M (1998). Maximum-likelihood estimation of population divergence times and population phylogeny in models without mutation. Evolution 52: 669-677.

Nordborg M (2000). Linkage disequilibrium, gene trees, and selfing: an ancestral recombination graph with partial selffertilisation. Genetics 154: 923-929.

Nordborg M (2001). Coalescent theory. In: Balding DJ, Bishop M, Cannings C (eds) Handbook of Statistical Genetics. Wiley: New York, pp 179-212.

Nordborg M, Tavaré S (2002). Linkage disequilibrium: what history has to tell us. Trends Genet 18: 83-90.

O'Ryan C, Harley EH, Bruford MW, Beaumont M, Wayne RK, Cherry MI (1998). Microsatellite analysis of genetic diversity in fragmented South African buffalo populations. Anim Conserv 1: 85-94

Overall ADJ, Nichols RA (2001). A method for distinguishing consanguinity and population substructure using multilocus genotype data. Mol Biol Evol 18: 2048-2056.

Parra EJ, Marcini A, Akey J, Martinson J, Batzer MA et al (1998). Estimating African American admixture proportions by use of population-specific alleles. Am J Hum Genet 63: 1839-1851.

Payseur BA, Nachman MW (2002). Gene density and human nucleotide polymorphism. Mol Biol Evol 19: 336-340.

Pluzhnikov A, Di Rienzo A, Hudson RR (2002). Inferences about human demography based on multilocus analyses of noncoding sequences. Genetics 161: 1209-1218.

Pritchard JK, Seielstad MT, Perez-Lezaun A, Feldman MW (1999). Population growth of human Y chromosomes: a study of Y chromosome microsatellites. Mol Biol Evol 16: 1791-1798.

Pritchard JK, Stephens M, Donnelly P (2000a). Inference of population structure using multilocus genotype data. Genetics 155: 945-959.

Pritchard JK, Stephens M, Rosenberg NA, Donnelly P (2000b). Association mapping in structured populations. Am J Hum Genet 67: 170-181.

Przeworski M, Hudson RR, Di Rienzo A (2000). Adjusting the focus on human variation. Trends Genet 16: 296-302.

Ptak SE, Przeworski M (2002). Evidence for population growth in humans is confounded by fine-scale population structure. Trends Genet 18: 559-563.

Rannala B, Hartigan JA (1996). Estimating gene flow in island populations. Genet Res Camb 67: 147-158.

Rannala B, Mountain JL (1997). Detecting immigration by using multilocus genotypes. Proc Natl Acad Sci USA 94: 9197-9201.

Reich DE, Goldstein DB (1998). Genetic evidence for a Paleolithic human population expansion in Africa. Proc Natl Acad Sci USA 95: 8119-8123.

Reich DE, Feldman MW, Goldstein DB (1999). Statistical properties of two tests that use multilocus data sets to detect population expansions. Mol Biol Evol 16: 453-466.

Relethford JH (2001). Genetic history of the human species. In: Balding DJ, Bishop M, Cannings C (eds) Handbook of Statistical Genetics. Wiley: New York, pp 813-846.

Rogers AR (1995). Genetic evidence for a Pleistocene population explosion. Evolution 49: 608-615.

Rogers AR (2001). Order emerging from chaos in human evolutionary genetics. Proc Natl Acad Sci USA 98: 779-780.

Rogers AR, Harpending H (1992). Population growth makes waves in the distribution of pairwise genetic differences. Mol Biol Evol 9: 552-569.

Rosenberg NA, Nordborg M (2002). Genealogical trees, coalescent theory and the analysis of genetic polymorphisms. Nat Rev Genet 3: 380-390.

Rosenberg NA, Pritchard JK, Weber JL, Cann HM, Kidd KK, Zhivotovsky LA et al (2002). Genetic structure of human populations. Science 298: 2381-2385.

Rousset F (2001). Inferences from spatial population genetics. In: Balding DJ, Bishop M, Cannings C (eds) Handbook of Statistical Genetics. Wiley: New York, pp 239-269. 
Saccheri IJ, Wilson IJ, Nichols RA, Bruford MW, Brakefield PM (1999). Inbreeding of bottlenecked butterfly populations: estimation using the likelihood of changes in marker allele frequencies. Genetics 151: 1053-1063.

Schlötterer C (2002). A microsatellite-based multilocus screen for the identification of local selective sweeps. Genetics 160: 753-763.

Shoemaker JS, Painter IS, Weir BS (1999). Bayesian statistics in genetics - a guide for the uninitiated. Trends Genet 15: 354-358.

Slatkin M, Hudson RR (1991). Pairwise comparisons of mitochondrial DNA sequences in stable and exponentially growing populations. Genetics 129: 555-562.

Smith BR, Herbinger CM, Merry HR (2001). Accurate partition of individuals into full-sib families from genetic data without parental information. Genetics 158: 1329-1338.

Stephens M (2001). Inference under the coalescent. In: Balding DJ, Bishop M, Cannings C (eds) Handbook of Statistical Genetics. Wiley: New York, pp 213-238.

Stephens M, Donnelly P (2000). Inference in molecular population genetics (with discussion). J R Stat Soc Ser B 62: 605-655.

Storz JF, Beaumont MA (2002). Testing for genetic evidence of population expansion and contraction: an empirical analysis of microsatellite DNA variation using a hierarchical Bayesian model. Evolution 56: 154-166.

Storz JF, Beaumont MA, Alberts SC (2002). Genetic evidence for long-term population decline in a savannah-dwelling primate: inferences from a hierarchical Bayesian model. Mol Biol Evol 19: 1981-1990.

Stringer CB, Andrews P (1988). Genetic and fossil evidence for the origin of modern humans. Science 239: 1263-1268.

Stumpf MPH, Goldstein DB (2001). Genealogical and evolutionary inference with the human Y chromosome. Science 291: $1738-1742$

Tajima F (1989). Statistical method for testing the neutral mutation hypothesis by DNA polymorphism. Genetics 123: 585-595.

Templeton AR (2002). Out of Africa again and again. Nature 416: 45-51.

Thompson EA (1973). The Icelandic admixture problem. Ann Hum Genet 37: 69-80.

Thomson R, Pritchard JK, Shen P, Oefner PJ, Feldman MW (2001). Recent common ancestry of human Y chromosomes: evidence from DNA sequence data. Proc Natl Acad Sci USA 97: 7360-7365.

Tishkoff SA, Varkonyi R, Cahinhinan N, Abbes S, Argyropoulos G, Destro-Bisol G (2001). Haplotype diversity and linkage disequilibrium at human G6PD: Recent origin of alleles that confer malarial resistance. Science 293: 455-462.

Vitalis R, Couvet D (2001). Estimation of effective population size and migration rate from one- and two-locus identity measures. Genetics 157: 911-925.
Vitalis R, Dawson K, Boursot P (2001). Interpretation of variation across marker loci as evidence of selection. Genetics 158: 1811-1823.

Wakeley J (1996). Distinguishing migration from isolation using the variance of pairwise differences. Theor Popul Biol 49: 369-386.

Wakeley J (1999). Nonequilibrium migration in human history. Genetics 153: 1863-1871.

Wakeley J (2001). The coalescent in an island model of population subdivision with variation among demes. Theor Popul Biol 59: 133-144.

Wakeley J, Nielsen R, Liu-Cordero SN, Ardlie K (2001). The discovery of single-nucleotide polymorphisms - and inferences about human demographic history. Am J Hum Genet 69: 1332-1347.

Wall JD (2000). A comparison of estimators of the population recombination rate. Mol Biol Evol 17: 156-163.

Wall JD, Przeworski M (2000). When did the human population size start increasing? Genetics 155: 1865-1874.

Wang D, Fan J, Siao C, Berno A, Young P, Sapolsky R et al (1998). Large-scale identification, mapping, and genotyping of single-nucleotide polymorphisms in the human genome. Science 280: 1077-1082.

Wang J (2001). A pseudo-likelihood method for estimating effective population size from temporally spaced samples. Genet Res Camb 78: 243-257.

Wang JL (2003). Maximum-likelihood estimation of admixture proportions from genetic data. Genetics 164: 747-765.

Whitlock MC, McCauley DE (1999). Indirect measures of gene flow and migration: $F_{\mathrm{ST}}$ not equal $1 /\left(4 N_{\mathrm{m}}+1\right)$. Heredity 82 117-125.

Williamson EG, Slatkin M (1999). Using maximum likelihood to estimate population size from temporal changes in allele frequencies. Genetics 152: 755-761.

Wilson GA, Rannala B (2003). Bayesian inference of recent migration rates using multilocus genotypes. Genetics 163 1177-1191.

Wilson IJ, Balding DJ (1998). Genealogical inference from microsatellite data. Genetics 150: 499-510.

Wilson IJ, Weale ME, Balding DJ (2003). Inferences from DNA data: population histories, evolutionary processes and forensic match probabilities. J Roy Stat Soc Ser A 166: 155-188.

Wilson JF, Weale ME, Smith AC, Gratrix F, Fletcher B, Thomas MG et al (2001). Population genetic structure of variable drug response. Nat Genet 29: 265-269.

Wright S (1931). Evolution in Mendelian populations. Genetics 16: $97-159$.

Wright S (1951). The genetic structure of populations. Ann Eugen 15: 323-354. 